\title{
Construction of Convergent Sequence in Cone 2-Normed Spaces
}

\author{
Sadjidon, Mahmud Yunus, and Sunarsini
}

\begin{abstract}
We introduce an idea of convergent sequence in a cone 2-normed space. We show that the convergence in 2normed spaces using the definition of 2 -norm by considering its dual space. Then we construct the convergence in cone 2 -normed space, particularly for $\ell_{2}$-space.
\end{abstract}

Index Terms-Convergent sequence, cone 2-normed spaces.

\section{INTRODUCTION}

$\mathbf{S}$ TUDY of 2-normed space continues to grow. Among others, the study of 2-norm is done by linking its dual space [1], especially for the $\ell_{2}$-space. With regards to the elaboration of the cone norm [2], [3], then the 2-norm was extended to cone 2-norm. Furthermore, the convergent properties in cone norm and 2-norm spaces have been studied in the literature. Then the construction of convergent sequence can be obtained on the cone 2-normed space, particularly for $\ell_{2}$-space. Furthermore, to construct the convergence in cone 2-normed space, first we discuss the 2-norm, 2-normed cone spaces and convergence criterion in 2-norm as follows.

Definition 1 ([4]). Let $X$ be a real vector space of dimension $d$, where $2 \leq d<\infty$. A 2-norm on $X$ is a function $\|\cdot, \cdot\|$ : $X \times X \rightarrow \mathbb{R}$ which satisfies:

(N1) $\|x, y\| \geq 0$ for every $x, y \in X ;\|x, y\|=0$ iff $x$ and $y$ are linearly dependent;

(N2) $\|x, y\|=\|y, x\|$ for every $x, y \in X$;

(N3) $\|x, \alpha y\|=|\alpha|\|x, y\|$ for every $x, y \in X$ and $\alpha \in \mathbb{R}$

(N4) $\|x, y+z\| \leq\|x, y\|+\|x, z\|$ for every $x, y, z \in X$.

The pair $(X,\|\cdot, \cdot\|)$ is called 2-normed space.

For history of inner product spaces and 2-normed spaces, we refer the interested reader to [4], [1], [5], [6], [7]. In [1], we have defined the 2-norm by linking its dual space with inner product $\langle x, z\rangle$, which is formally defined as

$$
\begin{aligned}
& \|x, y\|=\sup \left\{\begin{array}{ll}
\mid<x, z> & <y, z> \\
\mid<x, w> & <y, w>
\end{array} \mid ; z, w \in \ell^{2}\right. \\
& \text {; }\|z\|,\|w\| \leq 1\}
\end{aligned}
$$

Furthermore for every $x_{n}, x, y \in \ell_{2}$, we obtain

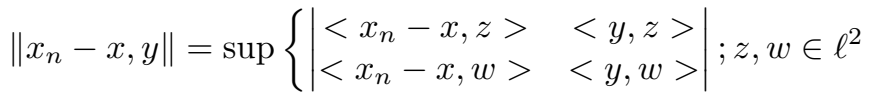

Manuscript received January 18, 2016; accepted January 20, 2016.

The authors are with the Department of Mathematics, Institut Teknologi Sepuluh Nopember, Surabaya 60111, Indonesia. Emails: \{djidon, yunusm, sunarsini\}@matematika.its.ac.id

This work has been supported by the Department of Mathematics ITS, by the Lembaga Penelitian dan Pengabdian pada Masyarakat ITS, by the General Directorate of Higher Education Indonesia (through Research Hibah).

$$
;\|z\|,\|w\| \leq 1\}
$$

Therefore for $n \rightarrow \infty$, then

$$
\sup \left\{\begin{aligned}
&\left|\begin{array}{ll}
<x_{n}-x, z> & <y, z> \\
<x_{n}-x, w> & <y, w>
\end{array}\right| ; z, w \in \ell^{2} \\
&;\|z\|,\|w\| \leq 1\}=0
\end{aligned}\right.
$$

Moreover for $m, n \rightarrow \infty$, then

$$
\begin{aligned}
& \sup \left\{\begin{array}{ll}
\mid<x_{m}-x_{n}, z> & <y, z> \\
<x_{m}-x_{n}, w> & <y, w>
\end{array} \mid ; z, w \in \ell^{2}\right. \\
& \text {; }\|z\|,\|w\| \leq 1\}=0
\end{aligned}
$$

Definition 2. A sequence $\left(x_{n}\right)$ in a 2-normed space $(X,\|\cdot, \cdot\|)$ is said to be convergent to $z \in X$ if for every $z \in X$ implies $\left\|x_{n}-x, z\right\|=0$ for $n \rightarrow \infty$. Also, we say that $\left(x_{n}\right)$ is Cauchy if for every $z \in X$ implies $\left\|x_{m}-x_{n}, z\right\|=0$ for $m, n \rightarrow \infty$.

Definition 3 ([3]). A cone normed space is an ordered pair $\left(X,\|\cdot\|_{C}\right)$ where $X$ is a linear space over $\mathbb{R}$ and $\|\cdot\|_{C}$ : $X \rightarrow(E, P,\|\cdot\|)$ is a function satisfying:

(C1) $\|x\|_{C} \geq \theta$ for every $x \in X$;

(C2) $\|x\|_{C}=\theta$ if and only if $x=0$;

(C3) $\|\alpha x\|_{C}=|\alpha|\|x\|_{C}$ for every $x \in X$ and $\alpha \in \mathbb{R}$;

(C4) $\|x+y\|_{C} \leq\|x\|_{C}+\|y\|_{C}$ for every $x, y \in X$;

It is easy to see that $\mathbb{R}^{n}$ equipped with the standard Euclidean norm is a Banach space and if $P \subset \mathbb{R}^{n}$ for nonnegative $\mathbb{R}, P$ is a cone.

Definition 4 ([3]). Let $X$ be a linear space over $\mathbb{R}$. Let $(E, \|$. I) be a Banach space and $P \subset E$ be a cone. If the function $\|\cdot, \cdot\|_{C}: X \times X \rightarrow(E, P,\|\cdot\|)$ satisfies

(CN1) $\|x, y\|_{C} \succeq \theta$ for every $x, y \in X ;\|x, y\|_{C}=$ iff $x$ and $y$ are linearly dependent;

(CN2) $\|x, y\|_{C}=\|y, x\|_{C}$ for every $x, y \in X$;

(CN3) $\|\alpha x, y\|_{C}=|\alpha|\|x, y\|_{C}$ for every $x, y \in X$ and $\alpha \in$ $\mathbb{R}$

(CN4) $\|x, y+z\|_{C} \preceq\|x, y\|_{C}+\|x, z\|_{C}$ for every $x, y, z \in$ $X$

then $\left(X,\|\cdot, \cdot\|_{C}\right)$ is called a cone 2-normed space.

\section{MAin Results}

Let $\ell_{2}$-space be a 2 -normed space. A function $\|\cdot, \cdot\|_{C}: \ell_{2} \times$ $\ell_{2} \rightarrow\left(\mathbb{R}^{n}, P,\|\cdot\|\right)$ defined by $\|x, y\|_{C}=\sum_{k=1}^{n} e_{k}\|x, y\|_{\ell_{2}}$, is a cone 2 -normed space and we say an $\ell_{2}$-space as a cone 2-normed space.

Theorem 1. Let $\left(\ell_{2},\left(\mathbb{R}^{n}, P,\|\cdot\|\right)\right)$ be a cone 2-normed space with $\|x, y\|_{C}=\sum_{k=1}^{n} e_{k}\|x, y\|_{\ell_{2}}$ and let $\left(x_{m}\right)$ be a sequence 
in $\ell_{2}$-space, then sequence $\left(x_{m}\right)$ is convergent to $x \in \ell_{2}$ if for every $z \in \ell_{2},\left\|x_{m}-x, z\right\|_{C}=\theta$ holds for $m \rightarrow \infty$.

Proof. The sequence $\left(x_{m}\right)$ converges to $x \in \ell_{2}$ implies that for every $z \in \ell_{2},\left\|x_{m}-x, z\right\|_{\ell_{2}}=0$ holds for $m \rightarrow \infty$. Moreover for every $z \in \ell_{2}$, then $\left\|x_{m}-x, z\right\|_{C}=\sum_{k=1}^{n} e_{k} \| x_{m}-$ $x, z \|_{\ell_{2}}$. Furthermore for $m \rightarrow \infty$ we have $\left\|x_{m}-x, z\right\|=$ $\sum_{k=1}^{n} e_{k}\left\|x_{m}-x, z\right\|_{\ell_{2}}=\sum_{k=1}^{n} e_{k} .0=\theta$. Therefore $\| z, x_{m}-$ $x \|_{C}=\theta$ for $m \rightarrow \infty$ and for every $z \in \ell_{2}$.

Theorem 2. Let $\left(\ell_{2},\left(\mathbb{R}^{n}, P,\|\cdot\|\right)\right)$ be a cone 2-normed space with $\|x, y\|_{C}=\sum_{k=1}^{n} e_{k}\|x, y\|_{\ell_{2}}$ and let $\left(x_{m}\right)$ be a sequence in $\ell_{2}$-space, then sequence $\left(x_{m}\right)$ is Cauchy if for every $z \in \ell_{2}$, $\left\|x_{m}-x, z\right\|_{C}=\theta$ holds for $m, n \rightarrow \infty$.

Proof. The sequence $\left(x_{m}\right)$ is Cauchy implies that for every $z \in \ell_{2},\left\|x_{m}-x_{n}, z\right\|_{l_{2}}=0$ holds for $m, n \rightarrow \infty$. Moreover for every $z \in \ell_{2}$, the following holds $\left\|x_{m}-x_{n}, z\right\|_{C}=$ $\sum_{k=1}^{n} e_{k}\left\|x_{m}-x_{n}, z\right\|_{l_{2}}$. Furthermore for $m, n \rightarrow \infty$, we have $\left\|x_{m}-x_{n}, z\right\|_{C}=\sum_{k=1}^{n} e_{k}\left\|x_{m}-x_{n}, z\right\|_{\ell_{2}}=\sum_{k=1}^{n} e_{k} \cdot 0=\theta$. Therefore $\left\|x_{m}-x_{n}, z\right\|_{C}=\theta$ for $m, n \rightarrow \infty$ and for every $z \in \ell_{2}$

Theorem 3. Let $\left(\ell_{2},\left(\mathbb{R}^{n}, P,\|\cdot\|\right)\right)$ be a cone 2-normed space with $\|x, y\|_{C}=\sum_{k=1}^{n} e_{k} N_{k}\|x, y\|_{\ell_{2}}$ for a nonnegative real number $N_{k}$ and let $\left(x_{m}\right)$ be a sequence in $\ell_{2}$-space, then sequence $\left(x_{m}\right)$ is convergent to $x \in \ell_{2}$ if for every $z \in \ell_{2}$, $\left\|x_{m}-x, z\right\|_{C}=\theta$ for $m \rightarrow \infty$.

Proof. The sequence $\left(x_{m}\right)$ converges to $x \in \ell_{2}$ implies that for every $z \in \ell_{2},\left\|x_{m}-x, z\right\|_{\ell_{2}}=0$ for $m \rightarrow \infty$. Moreover for every $z \in \ell_{2}$, the following holds $\left\|x_{m}-x, z\right\|_{C}=$ $\sum_{k=1}^{n} e_{k} N_{k}\left\|x_{m}-x, z\right\|_{\ell_{2}}$. Futhermore for $m \rightarrow \infty$, we have $\left\|x_{m}-x, z\right\|_{C}=\sum_{k=1}^{n} e_{k} N_{k}\left\|x_{m}-x, z\right\|_{\ell_{2}}=\sum_{k=1}^{n} e_{k} N_{k} .0=$ $\theta$. Therefore $\left\|z, x_{m}-x\right\|_{C}=\theta$ for $m \rightarrow \infty$ and for every $z \in \ell_{2}$.

Theorem 4. Let $\left(\ell_{2},\left(\mathbb{R}^{n}, P,\|\cdot\|\right)\right)$ be a cone 2-normed space with $\|x, y\|_{C}=\sum_{k=1}^{n} e_{k} N_{k}\|x, y\|_{\ell_{2}}$ for nonnegative real number $N_{k}$ and let $\left(x_{m}\right)$ be a sequence in $\ell_{2}$-space, then sequence $\left(x_{m}\right)$ is Cauchy if for every $z \in \ell_{2},\left\|x_{m}-x_{n}, z\right\|_{C}=\theta$ for $m, n \rightarrow \infty$.

Proof. The sequence $\left(x_{m}\right)$ is Cauchy implies that for every $z \in \ell_{2},\left\|x_{m}-x_{n}, z\right\|_{\ell_{2}}=0$ for $m, n \rightarrow \infty$. Moreover for every $z \in \ell_{2}$, the following holds $\left\|x_{m}-x_{n}, z\right\|_{C}=$ $\sum_{k=1}^{n} e_{k} N_{k}\left\|x_{m}-x_{n}, z\right\|_{\ell_{2}}$. Futhermore for $m, n \rightarrow \infty$, we have $\left\|x_{m}-x_{n}, z\right\|_{C}=\sum_{k=1}^{n} e_{k} N_{k}\left\|x_{m}-x_{n}, z\right\|_{\ell_{2}}=$ $\sum_{k=1}^{n} e_{k} N_{k} \cdot 0=\theta$. Therefore $\left\|x_{m}-x_{n}, z\right\|_{C}=\theta$ for $m, n \rightarrow \infty$ and for every $z \in \ell_{2}$.

Theorem 5. Let $\left(\ell_{2},\left(\mathbb{R}^{n}, P,\|\cdot\|\right)\right)$ be a cone 2-normed space and $\left(x_{m}\right)$ be a sequence in $\ell_{2}$ space, then sequence $\left(x_{m}\right)$ is convergent to $x \in \ell_{2}$ if for every $z \in \ell_{2},\left\|x_{m}-x, z\right\|_{C}=\theta$ for $m \rightarrow \infty$.

Proof. The sequence $\left(x_{m}\right)$ converges to $x \in \ell_{2}$ implies that for every $z \in \ell_{2},\left\|x_{m}-x, z\right\|_{\ell_{2}}=0$ holds for $m \rightarrow \infty$. Moreover for every $z \in \ell_{2}$, there exists a nonnegative real number $C_{k}$ such that $\left\|x_{m}-x, z\right\|_{C}=\sum_{k=1}^{n} e_{k} C_{k} \| x_{m}-$ $x, z \|_{\ell_{2}}$. Furthermore for $m \rightarrow \infty$, we have $\left\|x_{m}-x, z\right\|_{C}=$ $\sum_{k=1}^{n} e_{k} C_{k}\left\|x_{m}-x, z\right\|_{\ell_{2}}=\sum_{k=1}^{n} e_{k} C_{k} \cdot 0=\theta$. Therefore $\left\|z, x_{m}-x\right\|_{C}=\theta$ for $m \rightarrow \infty$ and for every $z \in \ell_{2}$.

Theorem 6. Let $\left(\ell_{2},\left(\mathbb{R}^{n}, P,\|\cdot\|\right)\right)$ be a cone 2-normed space and $\left(x_{m}\right)$ be a sequence in $l_{2}$-space, then sequence $\left(x_{m}\right)$ is Cauchy iffor every $z \in \ell_{2},\left\|x_{m}-x_{n}, z\right\|_{C}=\theta$ for $m, n \rightarrow \infty$.

Proof. The sequence $\left(x_{m}\right)$ is Cauchy implies for every $z \in \ell_{2}$, $\left\|x_{m}-x_{n}, z\right\|_{\ell_{2}}=0$ for $m, n \rightarrow \infty$. Moreover for every $z \in \ell_{2}$, with nonnegative real number $C_{k}$ such that $\left\|x_{m}-x_{n}, z\right\|_{C}=$ $\sum_{k=1}^{n} e_{k} C_{k}\left\|x_{m}-x_{n}, z\right\|_{\ell_{2}}$. Furthermore for $m, n \rightarrow \infty$, we have $\left\|x_{m}-x_{n}, z\right\|_{C}=\sum_{k=1}^{n} e_{k} C_{k}\left\|x_{m}-x_{n}, z\right\|_{\ell_{2}}=$ $\sum_{k=1}^{n} e_{k} C_{k} .0=\theta$. Therefore $\left\|x_{m}-x_{n}, z\right\|_{C} \rightarrow \theta$ for $m, n \rightarrow \infty$ and for every $z \in \ell_{2}$.

\section{CONCLUSIONS}

This work has developed convergence of a sequence in cone 2-normed spaces. This results can be extended to the convergence in $n$-normed space.

\section{REFERENCES}

[1] Sadjidon and H. Gunawan, "Konstruksi ruang 2-norm sebagai luasan yang direntang oleh dua vektor," Journal of Mathematics and Its Applications (LIMITS), vol. 4, no. 2, pp. 45-51, Aug. 2007.

[2] M. Eshaghi Gordji, M. Ramezani, H. Khodaei, and H. Baghani, "Cone normed spaces," Caspian Journal of Mathematical Sciences, vol. 1, no. 1, 2012.

[3] A. Sahiner and T. Yigit, "2-cone Banach spaces and fixed point theorem," AIP Conference Proceedings, vol. 1479, no. 1, pp. 975-978, 2012.

[4] H. Gunawan, "The space of p-summable sequences and its natural nnorm," Bulletin of the Australian Mathematical Society, vol. 64, no. 1, pp. 137-147, Aug. 2001.

[5] Sadjidon and Sunarsini, "Construction of difference cone normed space," in South East Asian Conference of Mathematics and Its Applications (SEACMA), 2013.

[6] Sadjidon, M. Yunus, and Sunarsini, "2-norma cone pada ruang $\ell_{2}$," in Simposium Nasional Analisis Matematika dan Aplikasinya (SNAMA), 2014.

[7] C. R. Diminnie, "A new orthogonality relation for normed linear spaces," Mathematische Nachrichten, vol. 114, no. 1, pp. 197-203, 1983. 